\title{
Sensor Context Information for Energy- Efficient Optimization of Wireless Procedures
}

\author{
Daniel Corujo ${ }^{1}$, Marcelo Lebre ${ }^{2}$, Diogo Gomes ${ }^{3}$, Rui L. Aguiar ${ }^{4}$ \\ Instituto de Telecomunicações \\ University of Aveiro \\ Aveiro, Portugal \\ $\left\{\right.$ dcorujo $^{1}$, marcelolebre ${ }^{2}$, dgomes $^{3}$, ruilaa $\left.{ }^{4}\right\} @$ av.it.pt
}

\begin{abstract}
The wide deployment of Wireless Local Area Networks (WLAN) we are witnessing today increases connectivity opportunities for mobile terminal devices, such as smartphones. However, continuous scanning for WLAN points of attachment can be a power exhausting mechanism for such battery-powered devices. These mobile devices, besides being equipped with different wireless access interfaces, are also coupled with sensors such as accelerometer, GPS, luminance and magnetic compass. In fact, sensors are increasingly being coupled into different devices and environments and are able to convey sensing information through networks into decision entities able to optimize different processes. In this paper we propose a framework where media independent sensing information is used to enhance wireless link management towards energy-efficiency. This framework enables the dissemination of sensing information towards local and remote decision entities, enhancing other processes (e.g. mobility) with sensing information in order to provide true Ambient Intelligence scenarios. We introduce this framework into a wireless management scenario able to provide energy-efficient optimal network connectivity.
\end{abstract}

Index Terms - Wireless Local Area Networks, Sensors, Energy-Efficient, Wireless Activation, IoT, Ambient Intelligence

\section{INTRODUCTION}

I $\mathrm{N}$ recent years, the increase of publicly available WLAN infrastructures has provided wireless-capable mobile devices with an alternative to other access technologies (e.g., $3 \mathrm{G}$ and HSDPA). To take advantage of these connectivity opportunities, mobile devices need to activate their wireless interfaces for scanning and attachment procedures, which highly impact energy consumption. However, these devices are also coming equipped with different kinds of sensors, which can aid in determining the best opportunity for activating specific wireless procedures. In fact, sensor devices are increasingly being coupled into different devices and scenarios. The evolution made over their capabilities and transmission technologies (e.g., 802.15.4 and Zigbee) has motivated the increase of off-the-shelf devices, encompassing a considerable range of different and diverse applications [1]. Sensors have became accessible to developers outside the sensor networks community and present a platform ready for software development, enabling the conception of Ambient Intelligent scenarios, embedding sensing input into different processes, such as industrial, entertainment and personal applications.

Accessing and disseminating such information is a prime objective when considering an Internet of Things, contributing to shortening the gap between our surrounding environments and the digital world at the same time that sensors are enabled to provide context information towards entities that are capable of consuming and using it to enhance other processes. However, this approach also introduces new problems. With the proliferation of sensor devices, manufacturers have supplied the market with different technologies, which operate distinctively and provide sensor information through very different formats. As such, sensing information frameworks have to resort to complex solutions in order to understand the different kinds of information and control the sensor devices.

With sensors deployed everywhere, constantly sending information through sensor and infrastructure networks in a multitude of scenarios, overall energy consumption will inevitably increase. This is currently a major concern for Information and Communication Technologies (ICTs), which have been mobilized by efforts such as the ICT for Energy Efficiency (ICT4EE ${ }^{1}$ ), aiming to facilitate the transition to an Energy-Efficient (EE) and low-carbon economy. We argue that sensors can provide context information, which can be used by ICTs (as well as other sectors) to improve EE processes, by using that information to optimize the operation of other processes, enabling them to be more efficiently controlled.

In this sense, we propose in this paper a framework that is able to integrate the information collected by different sensors, disseminates it towards consumers independently of the underlying transport technology, and which can act upon that information to optimize the EE of different processes. This framework is able to operate seamlessly in a bidirectional way, providing the means to control the sensors and configure how the information can be collected. We introduce this framework into a wireless management scenario, where the sensing information is used to improve EE of wireless

\footnotetext{
${ }^{1}$ http://www.ict4ee.eu/
} 
processes as well as to provide triggers for optimal network connectivity.

This paper is organized as follows. In Section II we present related work, followed by Section III where we introduce the architecture of our framework. In Section IV we submit our framework into an example scenario, first describing its integration with a EE wireless activation algorithm, following with results presentation. Finally, we conclude in Section V.

\section{RELATED WORK}

EE procedures have been a constant research topic in the evolution of wireless communications for decades now, providing different solutions at different levels of the stack over the years, comprising of physical, system and application level optimizations. However, single solutions are always reduced in scope, and when applied to cross-layer concerns, they present tradeoffs and inefficiencies. This has motivated methodologies combining not only link-layer $\mathrm{EE}$ improvements, but also other layer mechanisms such as [4] and [5]. The first considers adding a server with knowledge of the network workload to control the terminal's network card power configuration. In the second, a policy for network card selection based on power consumption is employed. Even though both present interesting energy savings, they are deployed in specific technologies (WLAN and Bluetooth) and make use of pre-designed policies working in specific scenarios, not considering sensors at all. We argue that a framework aimed at improving EE should be technologyagnostic, able to support different policies and support a large scope in terms of scenario deployment.

The deployment of sensors in ambient intelligence scenarios is typically associated to the wireless communication involved in reaching their sensing data. With the low-power characteristics of sensors, the power consumption used for wireless communication is a prime concern. When considering EE processes in wireless sensor networks, the typical approach is to focus on optimized wireless link protocols or energy conservation measures such as in [6]. Here, an optimized multi-hop sensor network is established showing that increasing the sensor node density in a wireless sensor network increases EE. However, solutions like these focus on the lower layers of the sensors, and the sensor network itself. This requires complex solutions when incorporating different technologies of sensors or integrating with high-level entities for sensor control and information usage in different scenarios.

The sensor information itself can play only a small role when it comes to providing it to complex higher-layer or application procedures, enabling their adaptation to dynamic environments. Several architectures exist with the purpose of collecting and distributing context information such as [7], featuring an agent-based architecture supporting contextaware systems, through a Context Broker. Other solutions, such as [8] rely on OSGi-based middleware. Both resort to XML information description which place higher processing and networking requirements, and require specific agents when sensors belong to different manufacturers. Also, [10] considers the provisioning of context as a service in heterogeneous environments, but the solution involves coupling to a specific set of high-level mechanisms and information representation, limiting deployment scenarios. This is where in fact our contribution aims to evolve, by providing a cross-layer approach for media-independent access to the control and information gathering from not only link interfaces but also sensorial devices, able to support different high-level mechanisms (e.g., mobility management) as well as allowing their integration in new scenarios, such as EE optimization.

\section{SENSOR CONTEXT INFORMATION FRAMEWORK}

In order to achieve $\mathrm{EE}$ wireless management procedures assisted by sensor information, our framework considers a set of goals. These are: media independent access to sensor information and control in heterogeneous sensor devices environments, the ability to affect wireless interface parameters and mechanisms for EE, and dissemination of the sensing information towards decision modules through a generic protocol able to work independently of the underlying transport technology.

To fulfill the first two goals, we have built our framework over the base concepts of 802.21 [2]. Here, a cross-layer entity (the Media Independent Handover Function - MIHF) abstracts link layers (accessed through link Service Access Points (SAP)) to high-level entities (here dubbed MIH-Users), via an abstract SAP called MIH_SAP. The MIHF has the ability to convey events, commands and information locally as well as remotely towards other MIHF-enabled entities, via the $\mathrm{MIH}$ Protocol, which can be transported over L2 or L3. In this way, both local and remote MIH-Users are able to receive information regarding the link interfaces of $\mathrm{MIH}$-enabled nodes (in the form of events) and issue commands over them based on their own specific algorithms.

For the third objective, we have enhanced the base 802.21 behavior by adding new events, commands and information allowing control and information-gathering able to be used independent of the different sensor technology. These extensions are assembled in the GENERIC_SENSOR_SAP, via simple adaptations that implement the MIH Protocol and interface with the MIHF, detailed in [3]. Figure 1 presents a high-level view of our framework, highlighting the base 802.21 components along our generic sensor extensions.

Equally important is that incorporating 802.21 services into our framework, allows us to control link interfaces for EE operations. More concretely, we take advantage of existing link commands to execute actions over the link behavior such as powering on or off the link, activate or deactivate wireless link activation, or even switching between passive and active scanning. Also, 802.21 allows the reutilization of the same signaling and decision mechanisms independently of the media used (e.g., WLAN, WiMAX or 3GPP), since the MIHF is able to abstract the commands and events from the MIHUsers towards the different link technologies, via their specific SAP. We retained that media independence by equally providing our sensor interactions in the form of a SAP. 


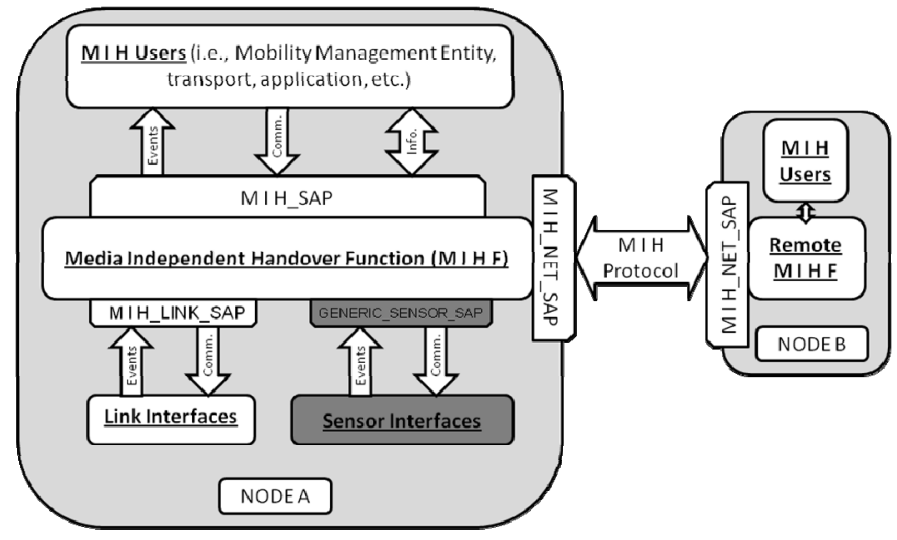

Figure 1 - Generic Sensor SAP Extension to 802.21

We present two deployment examples of our framework operation, depicted in Figure 2. In the first case, sensor devices within the mobile terminal are abstracted by the MIHF towards a local MIH-User, residing in the terminal. This MIHUser is able to use the sensor information as input towards EE decisions, using the MIH services to impact the wireless links for energy conservation. In the second case, the sensing information is conveyed to a MIH-User installed in a remote server, able to consider other network-related processes (e.g., mobility, Quality of Service, policies, etc.) or even the information provided by the sensors of multiple mobile terminals, and act on their wireless link interfaces for a global EE optimization procedure.

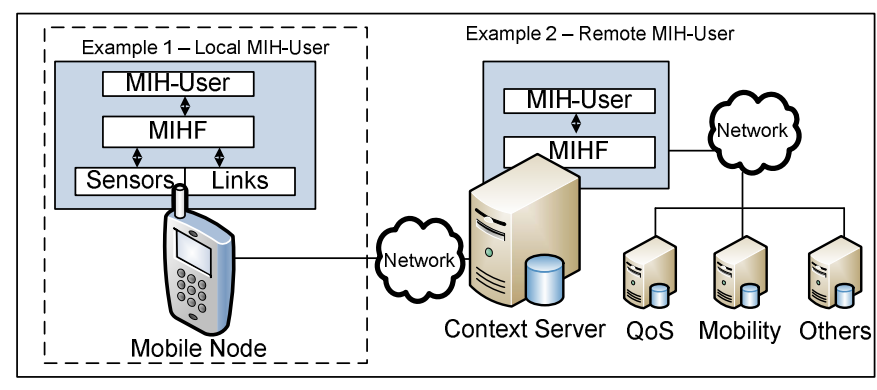

Figure 2 - Sensor Information Usage Scenarios

With the features provided by 802.21 , along with our generic sensor extensions towards collection and control of sensor devices in heterogeneous environments, we are able to provide the basis for, on one hand, integrate sensing information into existing processes such as mobility and, on the other, to provide a set of services that enable the abstraction of control and information gathering from different families and kinds of sensors, while enhancing them with media-independent service management mechanisms for node discovery, capability identification and service registration. We combine these possibilities towards sensor-aided EE wireless management procedures.

\section{Performance Evaluation}

Frameworks leveraging on sensors have to consider the limited processing, memory and network performance of such devices. With the abstraction capabilities provided by our framework, as well as the ability of conveying that information towards remote network decision entities, one might fear that sensor-related operations become burdened with the introduced mechanisms. Thus, it becomes necessary to assess the performance of our framework, even in very simple cases, but without withholding the amplitude of applications over which it can optimize procedures, particularly EE ones. As such, we developed and deployed a testbed scenario showing how a context-aware server can analyze media independent sensor information conveyed by a mobile terminal, and use such information to optimize EE capabilities on a multi-technology mobile terminal. Specific results on network performance using our developed signaling can be consulted in [3], whereas a study on the effect of our signaling in both node number and battery consumption can be found in [9].

\section{A. Energy-Efficient Sensor-Based Wireless Operation}

We setup a bi-partite testbed featuring two scenarios, depicted in Figure 3. The first scenario (the Base Run) was built for base comparison. Here, a mobile terminal with two Wi-Fi cards has one of them (wlan0) connected to AP1. The mobile user will approach AP2 and perform a handover using wlan 1, returning to AP1 when it leaves the vicinity of AP2. In this scenario, although a handover is performed, both Wi-Fi cards are active and continuously consuming energy. For scenario deployment simplicity, we feature two Wi-Fi cards, but the media independent mechanisms provided by our framework allow us to conceive multi-technology scenarios with little or no effort, simply by replacing these with interfaces belonging to different technologies, and enabling them with 802.21 support.

The second scenario (the Sensor Run) features a context server able to analyze media independent sensor events provided by the mobile terminal, issue remote commands accordingly and is also aware of the ESSID (Extended Service Set ID) for wireless networks in its vicinity for handover control. In this scenario, the mobile terminal is connected to AP1 using wlan0, and wlanl is powered down, conserving energy. When the mobile terminal moves (Movement 1), the accelerometer sensor sends a sensor event towards the context server, which processes it and remotely enables the wlan1 providing it with an ESSID for a closer AP (AP2), thus performing a quick attachment mechanism, saving on scanning time/energy. After attaching to AP2, the wlan0 is powered down, further conserving energy. On Movement 2), the opposite is occurs: as the mobile terminal departs from AP2, a sensor event sent to the context server triggers a command to power up wlan0, also providing the ESSID so it can directly attach to AP1, powering down wlan1 as well.

\section{1) Testbed}

In our testbed we separated the two APs by a distance of four meters, enabling the mobile terminal to move and experience signal strength changes from both APs. Our framework enables the setting of a threshold value that, when crossed, generates an event towards the context server with the information about the user's movement. 
Each scenario experiment has a duration of 10 minutes. In the first two minutes, the mobile terminal is close to AP1, then it moves to the AP2 and stays there for approximately 6 minutes, then returning to AP1 for another 2 minutes.

\section{2) Energy-Efficient Wireless Activation Algorithm}

Figure 4 represents the algorithm used in scenario 2, initiating its procedures after one interface becomes attached. The mobile terminal informs the context server when motion is detected, through a sensor event, which then sends a MIH command (MIH Link Action request), for powering up the inactive interface, along with the ESSID of a more suitable AP. Upon reception of this command, the mobile terminal powers up the inactive interface and attaches to the recommended AP. If this is unsuccessful, the terminal maintains its attachment to the current AP. In case of success, the previous interface will power down (thus conserving energy) and the mobile terminal will become attached to another interface. Although this is a simple handover algorithm, our scenario can be easily extended to encompass more complex features, such as full mobility protocols, using link events reporting radio conditions for optimizing handover opportunities or even able to interface with Authentication, Authorization and Accounting frameworks (AAA).

\section{3) Energy-Efficient Wireless Activation Architecture}

Figure 5 shows the message sequence diagram, depicting the message exchange by all entities present in the network. The used messages are sensor extensions implemented in ODTONE $^{2}$, an 802.21 open-source implementation, taking into consideration the messages defined in [3]. Initially, in step 0 we assume that the mobile terminal is already connected to an AP1. Step 1 represents the sensor event triggered by our Sensor SAP in the mobile terminal, which is sent to the MIHUser in the Context Server. Step 2 shows the triggered command flowing from the Context Server to the mobile terminal due to the movement generated by the mobile terminal while approaching AP2. This message promotes a handover from AP1 to AP2 (Step 3), powering up the inactive interface, while attaching it to AP2 and powering down the previously used interface connected to the AP1. Steps 4 to 6 highlight the opposite operation of steps 0 and 3 where the mobile terminal moves away from AP2 and approaches AP1 again.

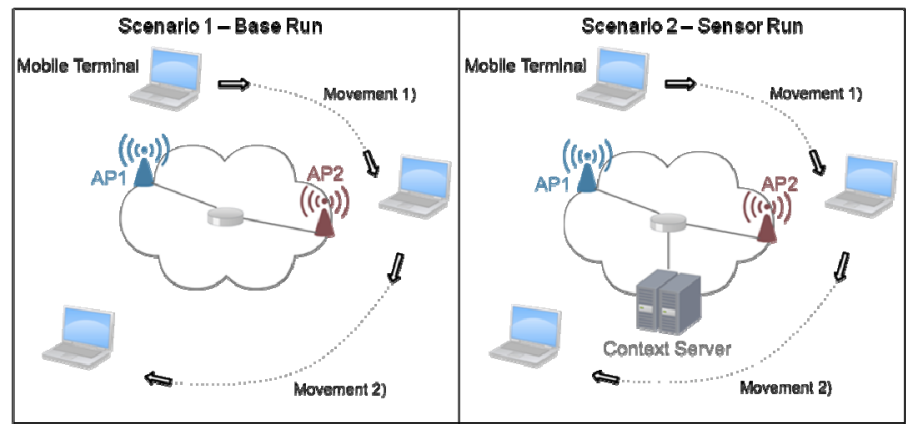

Figure 3 - Scenarios: Base Run (1) and Sensor Run (2)

${ }^{2}$ ODTONE - http://atnog.av.it.pt/projects/odtone

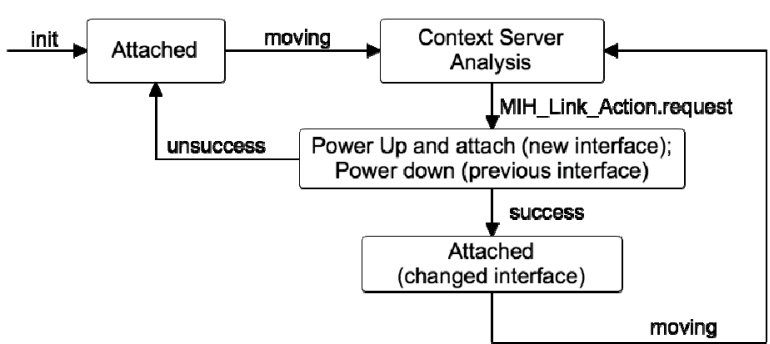

Figure 4 - Sensor Wireless Activation Algorithm

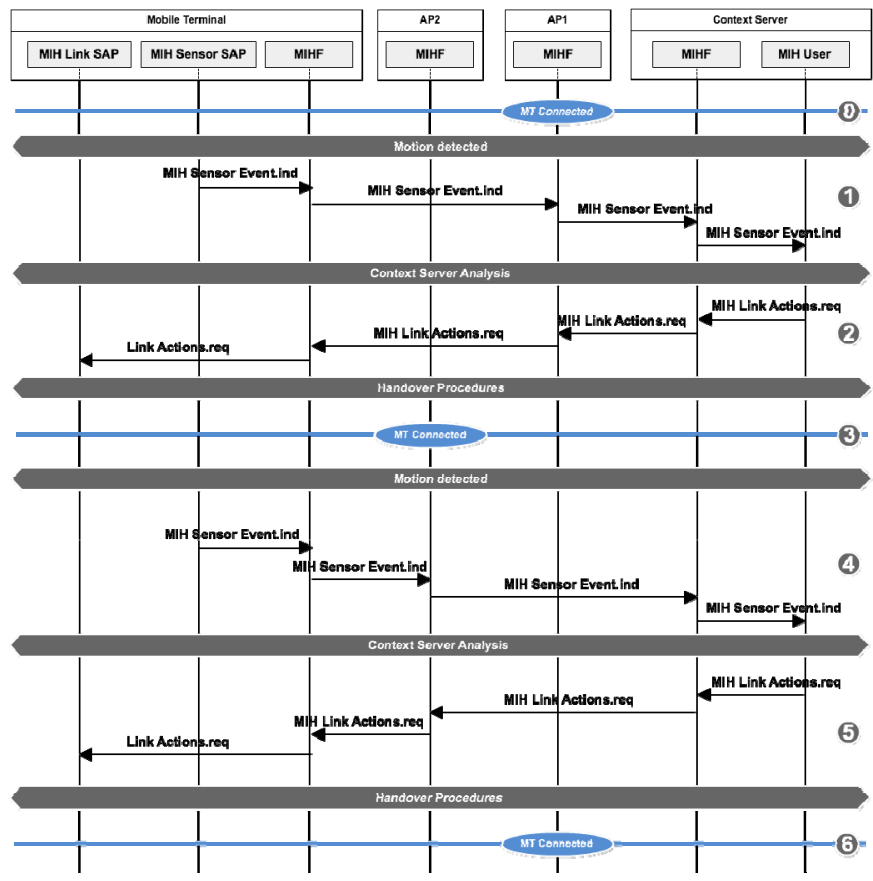

Figure 5 - Sequence Diagram for Wireless Activation

\section{B. Evaluation of Energy-Efficient Sensor-Based Wireless Operation}

To evaluate our framework we analyzed the energy consumption to assess the efficiency of optimizing wireless activation and how it would reflect on the mobile terminal's battery. The mobile terminal consists of a laptop with an Intel Centrino Duo processor, 2GB of RAM and two wireless cards. In this experiment we monitored the battery consumption continuously taking 2 second samples.

To analyze the energy consumption of each scenario we measured battery consumption for each scenario using the referred laptop. As the obtained measures were consistent, Figure 6 shows the comparison between two randomly chosen measures, one for each scenario, repeated for ten times.

Energy consumption was averaged in time intervals of 60 seconds during the 10 minutes duration of the scenario. This study shows that an average of $12.8 \mathrm{mWh}$ and $11.73 \mathrm{mWh}$ were consumed per sample for scenario 1 and 2 respectively.

The overall analysis provided by the ten repetitions on the battery consumption shows that scenario 1 has consumed an average total of $3784 \mathrm{mWh}$ of the battery's capacity and scenario 2 consumed $3579 \mathrm{mWh}$, representing an average saving of $200 \mathrm{mWh}$. 


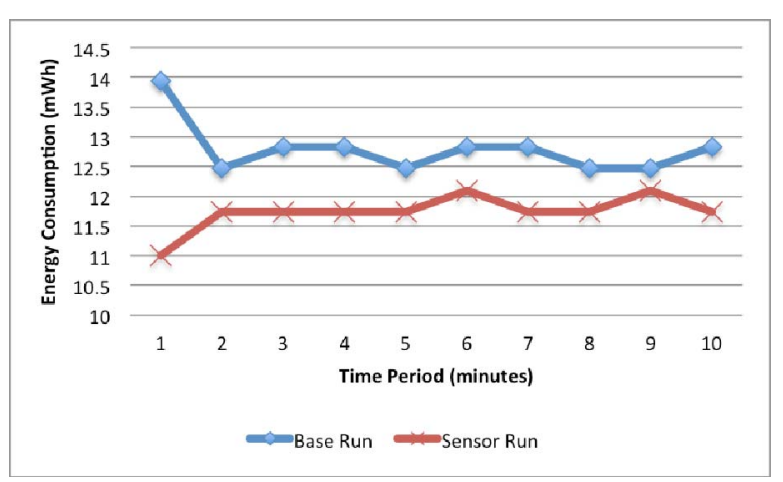

Figure 6 - Scenario Energy Consumption

\section{CONCLUSION}

In this paper we presented a framework where sensor information was added to the media independent mechanisms of the 802.21 standard, using that information to enable EE scenarios. Through a simple live experiment, we were able to convey sensor information obtained from a mobile terminal (enhanced with our media independent sensor-enabled mechanisms) towards a Context Server. This sensor information allowed decision mechanisms at the Context Server to control the activation/deactivation procedures of wireless interfaces of the mobile node for energy conservation, while providing pre-configured link information for optimal handover candidate selection and unnecessary wireless scanning avoidance. Even considering this simple deployment of our framework, we were able to evaluate the usefulness of sensor context information and achieve reduced power consumption at the terminal, even when such sensor information is conveyed remotely towards an external entity, such as the Context Server, for decision taking.

We argue that conveying sensor information obtained at mobile terminals towards network decision entities, enables them to take into consideration other contexts which are only obtainable from the mobile terminal's point of view, such as the energy requirements (and statuses) of mobile devices. These considerations can be furthered integrated with other procedures, such as optimal handover selection and decision, enabling network operators to fully optimize network management procedures (considering mobility, AAA, service optimization and other procedures), while also enabling the optimization of mobile terminal operations. By maintaining the structure and integrity of the MIH Protocol when extending it to contemplate sensor context information, we are not only enhancing the 802.21 mechanisms, but we are also allowing the integration of sensor information into any 802.21-enabled scenario, empowering handover decisions with more specialized elements, aiding in a more optimized process. This paper demonstrated a basic scenario, employing our framework over simple remote wireless activation/deactivation procedures while assisting in access point attachment, but these were already enough to obtain positive results on EE improvement.

Regarding future work, we are currently extending our scenarios and deploying our framework to more complex handover scenarios, considering different IP mobility protocols and decision algorithms. We are also implementing extended EE mechanisms over different wireless interfaces, beyond $\mathrm{Wi}-\mathrm{Fi}$, for a truly heterogeneous networks energyefficient sensor-aided environment. Lastly, we are also experimenting with sensors (and actuators) from different manufacturers, not only to further refine our sensor-enhanced 802.21 design, but also to integrate them into complex energyefficient ambient intelligence environment scenarios.

\section{REFERENCES}

[1] Akyildiz, I.F., Weilian Su, Sankarasubramaniam, Y., Cayirci, E., "A survey on sensor networks", IEEE Communications Magazine, pp 102113, vol. 40, Issue 8, November 2002

[2] IEEE 802.21 Standard, "Local and Metropolitan Area Networks - Part 21: Media Independent Handover Services", January 2009.

[3] Lebre, M., Corujo, D., Gomes, D., Aguiar, L., "Context Transport based on 802.21 MIH protocol", Proc. 1 CNRS 2011 - Conference on Wireless Sensor Networks, Coimbra, Portugal, Mar 2011

[4] Acquaviva, A., Simunic, T., Deolalikar, V. and Roy, S., "Remote power control of wireless network interfaces", J. Embedded Comput. 1, 3 (August 2005), 381-389.

[5] Qadeer, W., Rosing, T., Ankcorn, J., Krishnan, V. and De Micheli, G., "Heterogeneous Wireless Network Management", Lectures Notes in Computer Science, 2005, vol. 3164, pp.137-184.

[6] Rabaey, J.M.; Ammer, M.J.; da Silva, J.L., Jr.; Patel, D.; Roundy, S.; , " PicoRadio supports ad hoc ultra-low power wireless networking," Computer, vol.33, no.7, pp.42-48, Jul 2000

[7] H.Chen, "An intelligent broker architecture for pervasive ContextAware systems," Ph.D. dissertation, University of Maryland, Baltimore County, Dec. 2004.

[8] Gu, T.; Pung, H.K.; Zhang, D.Q.; , "Toward an OSGi-based infrastructure for context-aware applications," Pervasive Computing, IEEE , vol.3, no.4, pp. 66- 74, Oct.-Dec. 2004

[9] Corujo, D., Lebre, M., Gomes, D., Aguiar, L., "A Framework for Flexible Sensor Information Dissemination", Proc. 2nd International Workshop on Interconnections of Wireless Sensor Networks, Barcelona, Spain, Jun 2011

[10] Wagner, M.; Reichle, R.; Geihs, K.; , "Context as a service Requirements, design and middleware support," IEEE International Conference on Pervasive Computing and Communications Workshops (PERCOM),pp.220-225, March 2011 